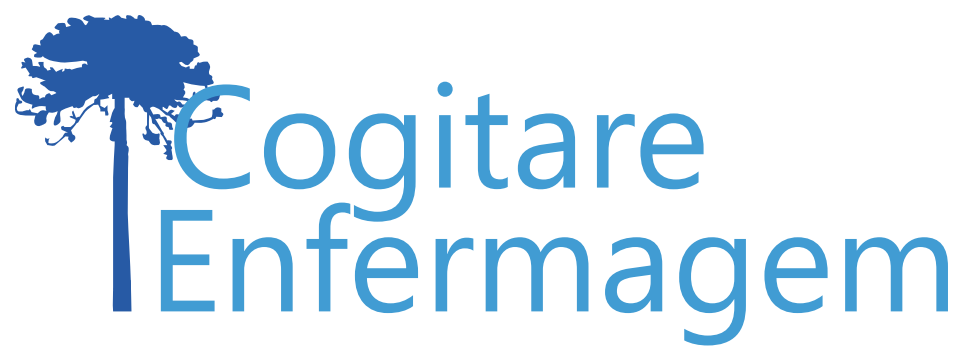

\title{
TRADUÇÃO E ADAPTAÇÃO TRANSCULTURAL DO INSTRUMENTO SELF CARE OF HEART FAILURE INDEX 6.2 PARA USO NO URUGUAI*
}

Paula Vanessa Peclat Flores ${ }^{1}$, Pablo Alvarez Rocha², Gabriela Mercedes Ormaechea ${ }^{3}$, Lyvia da Silva Figueiredo ${ }^{4}$, Nathália Sodré Velasco ${ }^{5}$ Ana Carla Dantas Cavalcanti ${ }^{6}$

\begin{abstract}
RESUMO
Objetivo: traduzir e adaptar transculturalmente o instrumento Self-Care of Heart Failure Index 6.2 para uso no Uruguai.

Método: estudo metodológico, realizado em clínica de cuidados multidisciplinares em insuficiência cardíaca no Uruguai entre novembro de 2016 e setembro de 2017. Foram desenvolvidas as etapas: tradução, síntese das versões traduzidas, retrotradução, avaliação pelo comitê de experts e préteste. Para análise, utilizou-se o índice de validade de conteúdo (adequado $>0,9$ ), percentual de concordância (adequado $\geq 80 \%$ ) e o alfa de Cronbach no pré-teste (adequado $\geq 0,70$ ).

Resultado: na primeira avaliação, seis itens apresentaram IVC $<0,9$ e/ou concordância total menor que $70 \%$ e por este motivo foram alterados e posteriormente reavaliados, atingindo IVC $>0,9$ e/ou percentual de concordâncias maior que $70 \%$. A consistência interna da escala foi de 0,78 .

Conclusão: a tradução e adaptação deste instrumento proporciona o desenvolvimento de estudos multicêntricos por proporcionar as mesmas medidas de avaliação de desfechos.
\end{abstract}

DESCRITORES: Autocuidado; Reprodutibilidade dos Testes; Tradução; Insuficiência Cardíaca; Pesquisa Metodológica em Enfermagem.

*Artigo extraído da tese de doutorado "Efeito da entrevista motivacional no autocuidado do paciente com insuficiência cardíaca: ensaio clínico randomizado". Universidade Federal Fluminense, 2018.

\section{COMO REFERENCIAR ESTE ARTIGO:}

Flores PVP, Rocha PA, Ormaechea GM, Figueiredo L da S, Velasco NS, Cavalcanti ACD. Tradução e adaptação transcultural do instrumento Self Care of Heart Failure Index 6.2 para uso no Uruguai. Cogitare enferm. [Internet]. 2019 [acesso em "colocar data de acesso, dia, mês abreviado e ano"]; 24. Disponível em: http:// dx.doi.org/10.5380/ce.v24i0.66326.

Este obra está licenciado com uma Licença Creative Commons Atribuição 4.0 Internacional.

${ }^{1}$ Enfermeira. Doutora em Ciências Cardiovasculares. Docente de Enfermagem e Pós-graduação em Enfermagem da Universidade Federal Fluminense. Niterói, RJ, Brasil. 9

${ }^{2}$ Médico. Professor Agregado da Facultad de Medicina. Hospital de Clinicas Manuel Quintela. Universidad de la Republica Uruguay. Coordenador da Unidad Multidisciplinaria de Insuficiencia Cardiaca. Montevidéo, Uruguay.

${ }^{3}$ Médica. Professora Agregada da Facultad de Medicina. Hospital de Clinicas Manuel Quintela. Universidad de la Republica Uruguay. Montevidéo, Uruguay.

${ }^{4}$ Enfermeira. Doutoranda em Ciências Cardiovasculares. Universidade Federal Fluminense. Niterói, RJ, Brasil. 9

${ }^{5}$ Enfermeira. Residente do Instituto Nacional do Câncer. Niterói, RJ, Brasil. 9

${ }^{6}$ Enfermeira. Docente de Enfermagem e Pós-graduação em Enfermagem da Universidade Federal Fluminense. Niterói, RJ, Brasil. () 


\title{
TRANSLATION AND TRANSCULTURAL ADAPTATION OF THE SELF- CARE INSTRUMENT OF HEART FAILURE INDEX 6.2 FOR USE IN URUGUAY
}

\begin{abstract}
Objective: To translate and cross-culturally adapt the Self-Care of Heart Failure Index 6.2 instrument for use in Uruguay.

Method: A methodological study, performed at a multidisciplinary heart failure care clinic in Uruguay between November 2016 and September 2017. The following stages were developed: translation, synthesis of translated versions, back-translation, expert committee evaluation and pre-test. For the analysis, the content validity index (adequate $>0.9$ ), agreement percentage (adequate $\geq 80 \%$ ) and Cronbach's alpha in the pre-test (adequate $\geq 0.70$ ) were used.

Result: In the first evaluation, six items displayed CVI $<0.9$ and/or a total agreement lower than $70 \%$ and for this reason were changed and subsequently reevaluated, reaching CVI > 0.9 and/or an agreement percentage greater than $70 \%$. The internal consistency of the scale was 0.78 .

Conclusion: The translation and adaptation of this instrument enables the development of multi-center studies by providing the same outcome assessment measures.
\end{abstract}

DESCRIPTORS: Self-care; Reproducibility of the Tests; Translation; Heart Failure Methodology in Nursing.

\section{TRADUCCIÓN Y ADAPTACIÓN TRANSCULTURAL DEL INSTRUMENTO SELF CARE OF HEART FAILURE INDEX 6.2 PARA SU USO EN URUGUAY}

\begin{abstract}
RESUMEN
Objetivo: traducir y adaptar transculturalmente el instrumento Self-Care of Heart Failure Index 6.2 para su uso en Uruguay.

Método: estudio metodológico, realizado en una clínica de cuidados multidisciplinares para insuficiencias cardíacas de Uruguay entre noviembre de 2016 y septiembre de 2017. Se desarrollaron las siguientes etapas: síntesis de las versiones traducidas, retrotraducción, evaluación a cargo de un comité de expertos y prueba previa. Para el análisis, se utilizó el índice de validez del contenido (adecuado $>0,9$ ), el porcentaje de concordancia (adecuado $\geq$ $80 \%$ ) y el coeficiente alfa de Cronbach en la prueba previa (adecuado $\geq 0,70$ ).

Resultado: en la primera evaluación, seis puntos presentaron un valor de IVC $<0,9$ y/o una concordancia inferior al $70 \%$ y, por este motivo, se los modificó y reevaluó posteriormente, con lo que se alcanzó un valor de IVC $>0,9$ y/o un porcentaje de concordancias superior al 70\%. La consistencia interna de la escala fue de 0,78.

Conclusión: la traducción y adaptación de este instrumento proporciona la capacidad para desarrollar estudios multicéntricos por ofrecer las mismas medidas de evaluación de resultados.
\end{abstract}

DESCRIPTORES: Autocuidado; Reproducibilidad de las pruebas; Traducción; Insuficiencia cardíaca; Investigación metodológica en Enfermería. 
A insuficiência cardíaca (IC) é importante problema de saúde mundial, que apresenta altas taxas de internação e mortalidade no mundo(1). Com população de 3,28 milhões de habitantes ${ }^{(2)}$, o Uruguai apresentou $27 \%$ de mortalidade por enfermidades cardiovasculares do total de óbitos em 2014, sendo a IC a terceira doença cardiovascular que mais internou no ano de $2013^{(3)}$.

O tratamento da IC é dividido em farmacológico e o não farmacológico ${ }^{(4-8)}$ e ambos dependem da participação ativa do paciente, já que sua capacidade de seguir o plano terapêutico e reconhecer sinais e sintomas de agravamento influencia no status de saúde ${ }^{(9)}$. O autocuidado são as condutas que os pacientes precisam seguir para manter sua saúde (manutenção do autocuidado), assim como o processo decisório sobre o possível agravo dos sintomas (gestão do autocuidado( ${ }^{(7)}$, sendo estas condutas determinantes no prognóstico ${ }^{(10)}$.

O estímulo ao autocuidado é elemento chave na educação do paciente e no autogerenciamento(11), uma vez que reconhecer a forma com que o paciente lida com o autocuidado frente à IC é estratégia vital para redimensionamento das orientações e condutas da equipe de saúde.

Dispor de instrumentos validados para mensurar o autocuidado oferece às equipes de saúde estratégia eficaz e de baixo custo para nortear seus cuidados, bem como otimiza a realização de estudos multicêntricos. Uma revisão sistemática identificou 14 instrumentos que mensuram o autocuidado e dentre eles, o Self-Care of Heart Failure Index (SCHFI) foi submetido a rigorosas provas psicométricas ${ }^{(12)}$ e avalia a manutenção, manejo e confiança no autocuidado. Criado nos Estados Unidos em 2000, em inglês americano, foi reajustado em 2009 chegando à versão SCHFI 6.2, adaptada para o chinês/Hong Kong(13), chinês/ Taiwan $^{(14)}$, italiano ${ }^{(15)}$, português/Brasil(16), português/Portugal ${ }^{(17)}$, japonês ${ }^{(18)}$, dentre outros.

É necessário que o instrumento esteja adequado às regionalidades da língua ${ }^{(19)}$ e o SCHFI 6.2 ainda não foi adaptado para uso no Uruguai. Este estudo se propôs a preencher esta lacuna, viabilizando a utilização do instrumento SCHFI 6.2 para uso em pacientes com insuficiência cardíaca em atendimento ambulatorial. O objetivo deste estudo foi traduzir e adaptar transculturalmente o instrumento Self-Care of Heart Failure Index 6.2 para uso no Uruguai.

\section{MÉTODO}

Estudo metodológico(20), destinado à tradução e adaptação transcultural(19) do SelfCare of Heart Failure Index 6.2 para utilização no Uruguai, desenvolvido entre novembro 2016 e outubro de 2017.

O SCHFI 6.2 possui 22 questões divididas em três escalas: Manutenção (10 itens), Manejo ( 6 itens) e Confiança no autocuidado ( 6 itens). As respostas para os itens variam de "nunca/raramente" a "sempre/diariamente" para escala de Manutenção do autocuidado; "pouco provável" a "muito provável" para escala de Manejo do autocuidado e "não confiante" a "extremamente confiante" para escala Confiança no autocuidado(16).

A manutenção do autocuidado está na sessão $A$ do instrumento e sua pontuação varia entre 10 e 40 pontos. Para chegar ao escore final, realiza-se o seguinte cálculo: (soma dos itens da sessão A-10)*3,333. Para o manejo do autocuidado (sessão B), a pontuação varia entre 4 e 24 pontos, e o escore é calculado através da (soma dos itens da sessão B-4)*5. Na sessão C, confiança no autocuidado, a pontuação varia entre 6 e 24 e o score é calculado com a (soma dos itens da sessão C-6)*5,56. Ẻm todas as subescalas, quanto maior o escore, melhor o resultado, sendo que o escore máximo, após o cálculo, é de 100 pontos em cada umas das escalas separadamente ${ }^{(16)}$. 
A primeira etapa da coleta de dados ocorreu em novembro de 2016 através do contato via e-mail com a autora principal solicitando consentimento para realização do estudo, o qual foi prontamente autorizado. O estudo foi desenvolvido nas seguintes etapas: tradução (por dois tradutores uruguaios juramentados), síntese das versões traduzidas, retrotradução (backtranslation), avaliação pelo comitê de experts e pré-teste ${ }^{(19)}$. As etapas de tradução e retrotradução ocorreram entre os meses de dezembro de 2016 e maio de 2017.

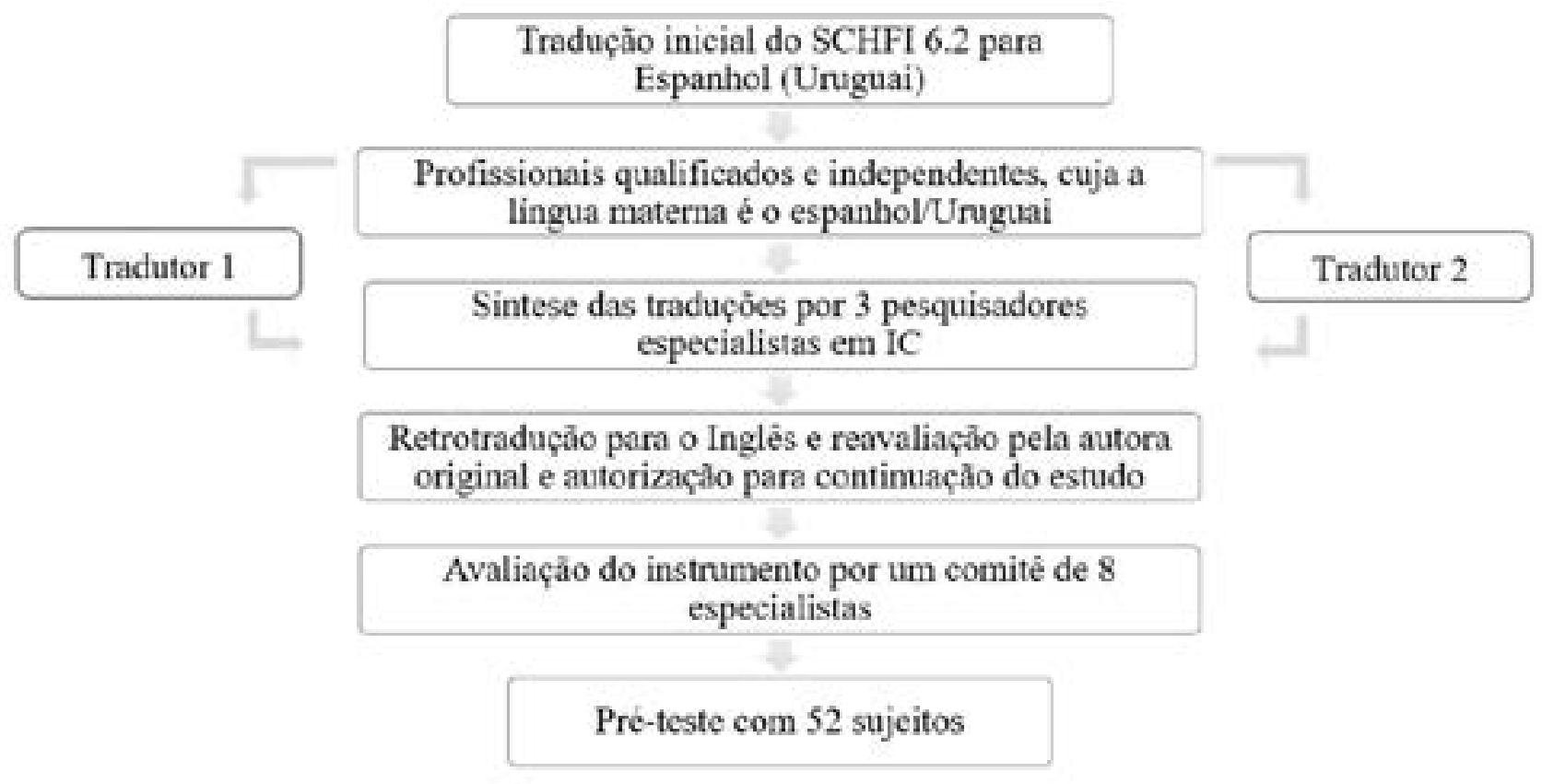

Figura 1 - Fluxograma do procedimento de adaptação transcultural do instrumento Self Care of Heart Failure Index 6.2. Rio de Janeiro, RJ, Brasil, 2019

O comitê de juízes foi composto por oito profissionais uruguaios com experiência profissional em ambulatório de IC (três médicos, dois nutricionistas, dois enfermeiros e um psicólogo), e iniciado em junho de 2017. Foi elaborado um instrumento com todos os itens da versão síntese do instrumento SCHFI 6.2 (autorizada pela autora original), no qual a equivalência semântica, idiomática experimental e conceitual foram avaliadas por uma escala Likert de 1 a 4 , considerando-se 1 'discordo plenamente', 2 'quase sempre discordo', 3 'concordo' e 4 'concordo plenamente'.

Após a avaliação do comitê de juízes atingir a versão final, iniciou-se o pré-teste, nos meses de agosto e setembro de 2017. Participaram desta etapa 52 pacientes, ou seja, número maior que o preconizado pelo Recommendations for the Cross-Cultural Adaptation of the DASH \& Quick DASH Outcome Measures ${ }^{(19)}$, que era de 30 pacientes. Foram incluídos pacientes em acompanhamento em uma clínica de cuidados multidisciplinares em IC, com mais de 18 anos, com diagnóstico prévio de IC. Estabeleceu-se como critério de exclusão relato de déficit cognitivo em prontuário ou avaliação clínica do psiquiatra da unidade, no entanto, nenhum dos incluídos apresentaram esta condição.

O instrumento SCHFI 6.2 na versão espanhol/Uruguai foi aplicado por um enfermeiro pesquisador, em consultório reservado, em um ambulatório especializado em insuficiência cardíaca no Uruguai, após o consentimento dos pacientes em participar do estudo. Dados sociodemográficos foram obtidos do prontuário eletrônico hospitalar.

Os dados foram organizados e analisados pelo Statistical Package for the Social 
Sciences-SPSS, versão 20.0. Foi utilizado índice de validade de conteúdo (IVC) e o percentual de concordância entre os juízes ${ }^{(21)}$. Considerou-se adequado o item com IVC $>0,9$ e/ou percentual de concordância total (item relevante ou representativo - 4) igual ou maior que $80 \%$. Os itens com avaliação inferior sofreram alterações, conforme sugestão dos juízes e foi realizada uma nova avaliação, sob os mesmos moldes da primeira(22-23). Utilizou-se - alfa de Cronbach para a análise da consistência interna e valores acima de 0,70 foram considerados alta consistência interna ${ }^{(24-25)}$.

As variáveis contínuas foram expressas através da média/desvio padrão ou mediana e intervalo interquartil. As variáveis categóricas foram expressas em frequências absolutas ou relativas (n e \%).

O estudo é uma das etapas de uma tese de doutoramento e está de acordo com os princípios da Declaração de Helsinki e decreto uruguaio 379/008 do Ministério de Saúde Pública(26), sendo aprovada pelo Comitê de Ética Médica do Hospital de Clínicas Manuel Quintela/Uruguai (17/05/2017).

\section{RESULTADOS}

\section{Tradução, síntese e retrotradução}

Inicialmente, a versão original do instrumento foi traduzida por dois tradutores uruguaios juramentados independentes. As duas versões traduzidas foram sintetizadas por dois pesquisadores uruguaios nativos no idioma. A versão síntese do instrumento foi retrotraduzida para o inglês por um terceiro tradutor e posteriormente enviada para autora principal do SCHFI 6.2, que autorizou o seguimento do estudo.

\section{Comitê de Juízes}

Na primeira avaliação, o comitê de juízes avaliou os itens da escala e seis itens (14, $21,22,23,24$ e 25) apresentaram IVC $<0,9$ e ou percentual de concordância total menor que $70 \%$, conforme os dados na Tabela 1.

Tabela 1 - Primeira avaliação dos juízes - percentual de concordância e índice de validade de Conteúdo (IVC). Rio de Janeiro, RJ, Brasil, 2019 (continua)

No ITEM

$\begin{array}{ccc}\begin{array}{c}\text { Quantidade } \\ \text { de }\end{array} & \begin{array}{c}\text { Percentual } \\ \text { de }\end{array} & \text { IVC } \\ \text { Avaliações } & \text { concordância } \\ \text { Represen- } & \% \\ \text { tativa (4) } & (\text { representa- } \\ (n=32) & \text { tivo/4) }\end{array}$

1 Para contestar las siguientes preguntas piense como se ha sentido durante el último mes o desde la última vez que nos vimos.

2 SECCIÓN A: A continuación, se enumeran instrucciones 31 97 1 comunes que se les da a las personas con insuficiencia cardíaca. ¿Con qué frecuencia usted hace lo siguiente?

\begin{tabular}{lllll}
\hline 3 & Nunca o rara vez & 31 & 97 & 1 \\
\hline 4 & Algunas veces & 31 & 97 & 1 \\
\hline
\end{tabular}


5 Frecuentemente

6 Siempre o diariamente

\begin{tabular}{ccc}
31 & 97 & 1 \\
\hline 32 & 100 & 1 \\
\hline 31 & 97 & 1 \\
\hline 27 & 84 & 1 \\
\hline 30 & 94 & 1
\end{tabular}

$7 \quad$ ¿Pesarse?

$8 \quad$ ¿Comprobar si tiene los tobillos hinchados?

9 ¿Prevenir enfermedades ( $p$. ej. vacunarse contra la gripe o evitar contacto con personas enfermas)?

10 ¿Hacer alguna actividad física?

\begin{tabular}{ccc}
28 & 88 & 1 \\
\hline 30 & 94 & 1 \\
\hline 24 & 75 & 1 \\
\hline 27 & 84 & 1 \\
\hline 7 & 22 & $\mathbf{0 , 3 4}$ \\
\hline 32 & 100 & 1 \\
\hline 27 & 84 & 1 \\
\hline 32 & 100 & 1
\end{tabular}

11 ¿Acudir a consultas con un médico o enfermero/a?

24

12 ¿Consumir una dieta con bajo contenido de sal?

24

13 ¿Hacer 30 minutos de ejercicio físico?

14 ¿Recordar tomar alguno de sus medicamentos?

15 ¿Pedir alimentos con bajo contenido de sal cuando sale a comer o visita a otras personas?

16 ¿Usar un sistema (pastillero, recordatorios) que lo ayude a recordar tomar su medicación?

17 SECCIÓN B: Muchos pacientes tienen síntomas debido a su insuficiencia cardiaca. La dificultad para respirar y los tobillos hinchados son síntomas comunes de la insuficiencia cardíaca.

18 En el pasado mes, ¿ha tenido dificultad para respirar o tobillos hinchados? Marque una opción: SI, No

19 Si ha tenido dificultad para respirar o tobillos hinchados en el último mes ... (marque un número con un círculo)

20 No tuve esto

(marque un número con un circulo)

\section{No lo reconocí}

\section{No muy rápido}

23 Un poco rápido

24 Rápido

27

29

29

27

27
16
11

84

0,97

25 Muy rápido

\begin{tabular}{ccc}
11 & 34 & 0,53 \\
\hline 8 & 25 & $\mathbf{0 , 5 9}$ \\
\hline 9 & 28 & $\mathbf{0 , 5 3}$ \\
\hline 17 & $\mathbf{5 3}$ & $\mathbf{0 , 5 3}$ \\
\hline 31 & 97 & 1 \\
\hline 30 & 94 & 1
\end{tabular}

27 En la continuación se enumeran medidas que utilizan las personas con insuficiencia cardíaca. Si usted tiene dificultad para respirar o tobillos hinchados, ¿qué probabilidad hay de que pruebe una de estas medidas? (marque un número con un círculo por cada medida)

\begin{tabular}{|c|c|c|c|c|}
\hline 28 & No es probable & 32 & 100 & 1 \\
\hline 28 & Poco probable & 32 & 100 & 1 \\
\hline 30 & Probable & 32 & 100 & 1 \\
\hline 31 & Muy probable & 31 & 97 & 1 \\
\hline 32 & Disminuir la sal en su dieta & 32 & 100 & 1 \\
\hline 33 & Disminuir la ingesta de líquidos & 30 & 94 & 1 \\
\hline 34 & Tomar un diurético extra & 31 & 97 & 1 \\
\hline 35 & Llamar a su médico o enfermero/a para pedir ayuda & 25 & 78 & 1 \\
\hline 36 & $\begin{array}{l}\text { Piense cual medida tomó la ultima vez que tuvo dificultad para } \\
\text { respirar o tobillos hinchados (marque un número con un círculo) }\end{array}$ & 30 & 94 & 1 \\
\hline
\end{tabular}




\begin{tabular}{|c|c|c|c|c|}
\hline 37 & No intenté nada & 25 & 78 & 1 \\
\hline 38 & No estaba seguro/a & 26 & 81 & 1 \\
\hline 39 & Algo seguro/a & 28 & 88 & 1 \\
\hline 40 & Seguro/a & 32 & 100 & 1 \\
\hline 41 & Muy seguro/a & 32 & 100 & 1 \\
\hline 42 & $\begin{array}{l}\text { ¿Qué tan seguro/a estaba de que la medida ayudaba o no } \\
\text { ayudaba? }\end{array}$ & 29 & 91 & 0,94 \\
\hline 43 & $\begin{array}{l}\text { SECCIÓN C: En general, ¿qué tan confiado está de poder hacer } \\
\text { lo siguiente? }\end{array}$ & 27 & 84 & 1 \\
\hline 44 & Nada confiado & 32 & 100 & 1 \\
\hline 45 & Algo confiado & 32 & 100 & 1 \\
\hline 46 & Muy confiado & 32 & 100 & 1 \\
\hline 47 & Extremadamente Confiado & 32 & 100 & 1 \\
\hline 48 & ¿Mantenerse sin síntomas de insuficiencia cardíaca? & 32 & 100 & 1 \\
\hline 49 & ¿Seguir las recomendaciones de tratamiento que le han dado? & 32 & 100 & 1 \\
\hline 50 & ¿Evaluar la importancia de sus síntomas? & 32 & 100 & 1 \\
\hline 51 & ¿Reconocer cambios en su salud si estos ocurren? & 31 & 97 & 1 \\
\hline & ¿Hacer algo para aliviar sus síntomas? & 32 & 100 & 1 \\
\hline 53 & ¿Evaluar qué tan bien funciona una medida? & 31 & 97 & 1 \\
\hline
\end{tabular}

Na seção A, a pergunta ¿Recordartomaralguno de sus medicamentos? apresentou IVC $=0,34$ e percentual de concordância $22 \%$ e recebeu três sugestões de alteração que foram consideradas na nova avaliação. Na seção B, cinco itens de resposta (no lo reconocí, no muy rápido, un poco rápido, rápido, muy rápido) receberam IVC e percentual de concordância fora do preconizado, no entanto, as sugestões dos peritos acarretaria incoerência com a pergunta "¿Qué tan rápido reconoció esto como un síntoma de insuficiencia cardíaca?", neste sentido, foram elaboradas duas opções de perguntas.

A Tabela 2 descreve a segunda avaliação dos juízes, sendo escolhidos os itens que apresentaram maior IVC e percentual de concordância. Na seção A, a opção 3 " ¿Ha olvidado tomar alguno de sus medicamentos?" , foi melhor avaliada, apresentando valores máximos de IVC e percentual de concordância. Na secção B, a opção 2 apresentou melhores índices de IVC e percentual de concordância, e será incluída na SCHFI 6.2 espanhol/Uruguai.

Tabela 2 - Segunda avaliação dos juízes - percentual de concordância e índice de validade de Conteúdo (IVC). Rio de Janeiro, RJ, Brasil, 2019 (continua)

\section{ITEM}

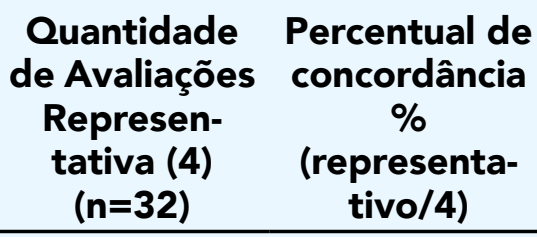

IVC

\begin{tabular}{|c|c|}
\hline $\begin{array}{l}\text { de Avaliações } \\
\text { Represen- } \\
\text { tativa (4) } \\
(n=32)\end{array}$ & $\begin{array}{c}\text { concordância } \\
\% \\
\text { (representa- } \\
\text { tivo/4) }\end{array}$ \\
\hline
\end{tabular}

SECÇÃO A

OPICIÓN 1.: ¿Recuerda tomar los medicamentos como se lo

28

88

0,88

indicó el médico? 
OPICIÓN 2.: ¿Olvida tomar alguno de sus medicamentos?

OPICIÓN 3.: ¿Ha olvidado tomar alguno de sus

24

88

32

100

1 medicamentos?

SECÇÃO B

OPÇÃO 1

¿Cuánto demoró en darse cuenta que eso era un síntoma de insuficiencia cardiaca?

\begin{tabular}{llcc}
\hline No me di cuenta & 29 & 91 & 1 \\
\hline Demoré mucho & 27 & 84 & 1 \\
\hline Demoré bastante & 24 & 75 & 0,94 \\
\hline Demore un poco & 20 & 63 & 0,84 \\
\hline Nada & 27 & 84 & 0,97 \\
\hline Médias da opção 1 & 155 & 81 & 0,96 \\
\hline OPÇÃO 2 & & & \\
\hline ¿ué tan rápido se dio cuenta que eso era un síntoma de & 32 & 100 & 1 \\
insuficiencia cardiaca? & & & 100 \\
\hline No me di cuenta & 32 & 75 & 0,91 \\
\hline Bastante tarde & 24 & 84 & 0,97 \\
\hline Un poco tarde & 27 & 97 & 1 \\
\hline Enseguida & 31 & 100 & 1 \\
\hline De imediato & 32 & $\mathbf{9 3}$ & $\mathbf{0 , 9 8}$
\end{tabular}

\section{Pré-teste}

Para o pré-teste, 52 pacientes responderam a versão do questionário de autocuidado 6.2 Espanhol/Uruguai, conforme apresentado na Tabela 3. Ressalta-se que o manejo de autocuidado só é avaliado nos pacientes que apresentaram falta de ar e/ou edema de membros inferiores nos últimos 30 dias, sendo assim, neste item a amostra se restringiu a 21 pacientes.

Tabela 3 - Caracterização sociodemográfica, clínica e de autocuidado ( $n=52)$. Rio de Janeiro, RJ, Brasil, 2019 (continua)

\begin{tabular}{lc} 
Variáveis & $\mathbf{n = 5 2}$ \\
\hline Idade, anos $^{\star}$ & $64 \pm 13$ \\
\hline Gênero masculino $^{\dagger}$ & $30(57,7)$ \\
\hline${\text { Peso, } \mathrm{kg}^{\star}}^{\text {Vivendo com a familia }^{\dagger}}$ & $87,7 \pm 23,4$ \\
\hline Classe funcional da New York Heart Association $^{\dagger}$ & $31(59,6)$ \\
\hline I & $18(34,6)$ \\
\hline
\end{tabular}




\begin{tabular}{lc}
\hline II & $20(38,4)$ \\
\hline III & $7(13,5)$ \\
\hline Sem avaliação de classe funcional & $7(13,5)$ \\
\hline Autocuidado & \\
\hline Manutenção* & $63,13 \pm 15,15$ \\
\hline Manejo* & $44,52 \pm 23,01$ \\
\hline Confiança* & $72,27 \pm 24,29$ \\
*Média/Desvio Padrão; ${ }^{\text {Frequência Simples (percentual) }}$
\end{tabular}

\section{Consistência Interna}

Utilizou-se valores do alfa de Cronbach de outros estudos de tradução/adaptação transcultural realizados com a SCHFI para analisar os resultados obtidos neste estudo, conforme a Tabela 4.

Tabela 4 - Comparação entre as análises da consistência interna de diversas línguas da escala SCHFI 6.2, através do alfa de Cronbach. Rio de Janeiro, RJ, Brasil, 2019

\begin{tabular}{|c|c|c|c|c|}
\hline País & Manutenção & Manejo & Confiança & Total \\
\hline Uruguai & 0,55 & 0,93 & 0,91 & 0,78 \\
\hline Inglês $(6.2 / 2009)^{(27)}$ & 0,55 & 0,59 & 0,82 & $-*$ \\
\hline Inglês $(2004)^{(29)}$ & 0,56 & 0,7 & 0,82 & $-\star *$ \\
\hline Brasil $^{(16)}$ & 0,43 & 0,76 & 0,94 & 0,77 \\
\hline China $^{(13)}$ & $-*$ & $-*$ & $-*$ & 0,73 \\
\hline Taiwan $^{(14)}$ & 0,63 & 0,71 & 0,86 & - \\
\hline Italiano(15) & 0,56 & 0,7 & 0,82 & 0,76 \\
\hline Portuga| $\left.\right|^{(17)}$ & 0,64 & 0,57 & 0,84 & $-*$ \\
\hline Japão(18) & 0,68 & 0,58 & 0,87 & $-\star$ \\
\hline
\end{tabular}

*Valores não identificados nos estudos publicados.

\section{DISCUSSÃO}

Este foi o primeiro estudo desenvolvido para a tradução e adaptação de uma escala para avaliar o autocuidado de pacientes com insuficiência cardíaca para uso no Uruguai. Os pacientes incluídos no pré-teste apresentaram características sociodemográficas similares de outros estudos de elaboração, tradução e adaptação do SCHFI 6.2 ${ }^{(13-18,27,28)}$.

O estudo apresentou alto grau de concordância entre os peritos, indicando de forma expressiva quais eram os seis itens que necessitavam de reestruturação. Em um dos casos, estes itens estavam vinculados ao ato de 'recordar' ou 'não recordar' e no outro item, se relacionava a uma avaliação de um ato subjetivo como é a percepção dos sintomas. Neste 
sentido, acreditamos que a experiência dos juízes no manejo de pacientes com IC foi de grande influência, além do conhecimento do modo linguístico que os pacientes utilizam para se referir aos sintomas e aos hábitos de autocuidado.

Especificamente nos itens de resposta na seção B, observa-se que as sugestões dos juízes levaram à modificação de respostas, que implicariam num conceito de agilidade (no muy rapido, um poco rapido, rapido, muy rápido) em relação à resposta que permite ao paciente a manejar seu autocuidado, com uma concepção temporal tangível (bastante tarde, um poco tarde, enseguida, de imediato).

Em relação à tomada de medicação(10), é provável uma questão idiossincrática da população uruguaia para dar mais peso ao evento negativo 'de esquecer', do que positivo 'de recordar', no entanto, essa é uma informação difícil de verificar neste estudo. Em qualquer caso, o importante foi o alto índice de concordância entre os juízes acerca da necessidade de modificar este item, assim como na aceitação da sua reformulação.

A adesão ao tratamento medicamentoso, a participação às consultas e consumo de dieta com baixo sal(4-8,10), foram os itens com melhor escore dos pacientes. A adesão à dieta hipossódica geralmente é uma medida de autocuidado de baixa adesão(7), no entanto, o Ministério da Saúde do Uruguai possui um programa de incentivo à diminuição do consumo de sal, que é fortemente defendido e aplicado na clínica de insuficiência cardíaca no qual se realizou este estudo, e com isso já apresenta resultados positivos.

O gerenciamento do autocuidado está diretamente relacionado a ações autônomas, diretamente relacionadas à prévia orientação da equipe de saúde; inicia-se com a interpretação de sintomas (medido pelo Item 11), reduzindo o sal na dieta (Item 12) e reduzindo a ingestão de líquidos ${ }^{(15)}$.

Há uma controvérsia na literatura acerca dos valores de referência para o alfa de cronbach, mas é possível questionar o motivo pelo qual todos os estudos não apresentaram coeficientes mais elevados. A SCHFI não é uma escala unidimensional, um exemplo claro disso é que na escala de manutenção do autocuidado, temos mais de uma dimensão de autocuidado representada, como seguir uma dieta adequada, realizar atividades físicas, ingerir as medicações corretamente. Os itens são homogêneos dentro de cada cuidado (dieta, medicação, presença nas consultas), no entanto, na escala como um todo, eles podem apresentar-se heterogeneamente ${ }^{(29)}$.

Percebe-se que na escala de manutenção do autocuidado, o alfa de cronbach foi similar em todos os estudos citados ${ }^{(13-18,27-28)}$. Já na escala de manejo, este estudo apresentou índices superiores a outros estudos, o que pode estar relacionado com a pouca divergência entre as respostas encontradas. Isto pode estar relacionado ao estilo do serviço e maneira como conduzem as orientações para manejo da doença.

O estudo limita-se pelo fato de ter sido realizado em um único centro, que embora seja o cento de referência em IC do país, recebendo pacientes de diversas regiões, não representa a população como um todo.

\section{CONSIDERAÇÕES FINAIS}

Este estudo traduziu e adaptou o SCHFI 6.2 para avaliação do autocuidado de pacientes com Insuficiência Cardíaca no Uruguai, adotando o nome "Índice de Autocuidado en la Insuficiência Cardíaca (Uruguai)". A versão traduzida e adaptada apresentou consistência interna satisfatória, similar aos demais estudos de tradução/adaptação transcultural da mesma escala, realizados em diversos países.

A tradução e adaptação deste instrumento para uso no Uruguai é essencial no ponto de vista clínico e acadêmico, uma vez que a presença de escalas adaptadas proporciona avaliação mais precisa dos pacientes e favorecem desenvolvimento de estudos 
multicêntricos, podendo assim, utilizar as mesmas medidas de avaliação de desfechos. Este instrumento está disponível gratuitamente para download em: http://self-care-measures. com/project/patient-version-schfi-uruguay/.

\section{AGRADECIMENTOS}

À autora original do instrumento SCHFI 6.2, pela autorização para realização da tradução e adaptação transcultural do mesmo, à Universidade Santander, que através da Bolsa Fórmula Santander apoiou a internacionalização envolvida neste estudo (aprovado em 20 de dezembro de 2016) e à Fundação de Amparo à Pesquisa do Estado do Rio de Janeiro/FAPERJ, que apoiou a publicação deste estudo.

\section{REFERÊNCIAS}

1. Bressler J, Knopman DS, Sharrett AR, Gottesman RF, Penman A, Chang PP, et al. Incident heart failure and cognitive decline: the atherosclerosis risk in communities study. J Card Fail [Internet]. 2017 [acesso em 20 abr 2019]; 23(1). Disponível em: https://doi.org/10.1016/j.cardfail.2016.11.002.

2. República Oriental del Urugay. Instituto Nacional de Estadística. Departamento de Difusíon y Comunicacíon. Anuario Estadístico 2018. [Internet]. 2018 [acesso em 12 jul 2019]. Disponível em: http:// www.ine.gub.uy/anuario-estadistico.

3. Comisión Honoraria para la Salud Cardiovascular- Área de Epidemiología y Estadística. Mortalidad por enfermedades cardiovasculares en el Uruguay 2014-2013. [Internet]. 2014 [acesso em 22 abr 2019]. Disponível em: http://www.cardiosalud.org/files/documents/morbilidad 2013 y mortalidad 2014.pdf.

4. Yancy CW, Jessup M, Bozkurt B, Butler J, Jr Casey DE, Colvin MM, et al. 2017 ACC/AHA/HFSA focused update of the 2013 ACCF/AHA guideline for the management of heart failure: a report of the American College of Cardiology/American Heart Association Task Force on Clinical Practice Guidelines and the Heart Failure Society of America. Circulation [Internet]. 2017 [acesso em 20 abr 2019]; 136(6). Disponível em: https://doi.org/10.1161/CIR.0000000000000509.

5. Ponikowski P, Voors AA, Anker SD, Bueno H, Cleland JGF, Coats AJS, et al. 2016 ESC Guidelines for the diagnosis and treatment of acute and chronic heart failure: The Task Force for the diagnosis and treatment of acute and chronic heart failure of the European Society of Cardiology (ESC) Developed with the special contribution of the Heart Failure Association (HFA) of the ESC. Eur Heart J [Internet]. 2016 [acesso em 21 abr 2019]; 37(27). Disponível em: https://doi.org/10.1093/eurheartj/ehw128.

6. Rohde LE, Montera MW, Bocchi EA, Clausell N, Albuquerque DC de, Rassi S, et al. Diretriz Brasileira de Insuficiência Cardíaca Crônica e Aguda. Arq Bras Cardiol [Internet]. 2018 [acesso em 22 abr 2019]; 111(3). Disponível em: http://publicacoes.cardiol.br/portal/abc/portugues/2018/v11103/pdf/11103021.pdf.

7. Conceição AP da, Santos MA dos, Santos B dos, Cruz DALM da. Self-care in heart failure patients. Rev. Latino-Am. Enfermagem [Internet]. 2015 [acesso em 22 abr 2019]; 23(4). Disponível em: http://dx.doi. org/10.1590/0104-1169.0288.2591.

8. Tawalbeh LI, Qadire MA, Ahmad MM, Aloush S, Sumaqa YA, Halabi M. Knowledge and self-care behaviors among patients with heart failure in Jordan. Res Nurs Health [Internet]. 2017 [acesso em $22 \mathrm{abr}$ 2019]; 40(4). Disponível em: https://doi.org/10.1002/nur.21805.

9. Keteyian SJ, Squires RW, Ades PA, Thomas R. Incorporating patients with chronic heart failure into outpatient cardiac rehabilitation: Practical recommendations for exercise and self-care counseling - $A$ clinical review. J Cardio Rehabil Prev [Internet]. 2014 [acesso em 22 abr 2019]; 34 (4). Disponível em: https://doi.org/10.1097/HCR.0000000000000073. 
10. Saccomann ICR da S, Cintra FA, Gallani MCBJ. Factors associated with beliefs about adherence to non-pharmacological treatment of patients with heart failure. Rev Esc Enferm USP [Internet]. 2014 [acesso em 22 abr 2019]; 48(1). Disponível em: http://dx.doi.org/10.1590/S0080-623420140000100002.

11. Kristiansen AM, Svanholm JR, Schjødt I, Jensen KM, Silén C, Karlgren K. Patients with heart failure as co-designers of an educational website: implications for medical education. Int J Med Educ [Internet]. 2017 [acesso em 22 abr 2019]; 8. Disponível em: http://doi.org/10.5116/ijme.5898.309e.

12. Cameron J, Worrall-Carter L, Driscoll A, Stewart S. Measuring self-care in chronic heart failure: a review of the psychometric properties of clinical instruments. J Cardiovasc Nurs [Internet]. 2009 [acesso em 22 abr 2019]; 24(6). Disponível em: http://doi.org/10.1097/JCN.0b013e3181b5660f.

13. Yu DSF, Lee DTF, Thompson DR, Woo J, Leung E. Assessing self-care behaviour of heart failure patients: cross-cultural adaptation of two heart failure selfcare instruments. Hong Kong Med J [Internet]. 2010 [acesso em 22 abr 2019]; 16(Suppl 3). Disponível em: https://www.hkmj.org/system/files/ hkm1006sp3p13.pdf.

14. Tung HH, Chen SC, Yin WH, Cheng CH, Wang TJ, Wu SF. Self-care behavior in patients with heart failure in Taiwan. Eur J Cardiovasc Nurs [Internet]. 2012 [acesso em 22 abr 2019]; 11(2). Disponível em: https://doi.org/10.1016/j.ejcnurse.2011.02.002.

15. Vellone E, Riegel B, Cocchieri A, Barbaranelli C, D'Agostino F, Antonetti G, et al. Psychometric testing of the self-care of heart failure index version 6.2. Res Nurs Health [Internet]. 2013 [acesso em $22 \mathrm{abr}$ 2019]; 36(5). Disponível em: https://doi.org/10.1002/nur.21554.

16. Ávila CW, Riegel B, Pokorski SC, Camey S, Silveira LCJ, Rabelo-Silva ER. Cross-cultural adaptation and psychometric testing of the brazilian version of the Self-Care of Heart Failure Index version 6.2. Nurs Res Pract [Internet]. 2013 [acesso em 22 abr 2019]; 2013. Disponível em: http://dx.doi. org/10.1155/2013/178976.

17. Marques CP, Lopes MJ, Rebola E, Pequito T. Self-care in Patients with Heart Failure. RIASE [Internet]. 2016 [acesso em 23 abr 2019]; 2(1). Disponível em: http://dx.doi.org/10.24902/r.riase.2016.2(1).439.

18. Kato N, Kinugawa K, Nakayama E, Tsujl T, Kumagai Y, Miura C, et al. Psychometric testing of the Japanese Version of the Self-Care of Heart Failure Index. J Card Fail [Internet]. 2013 [acesso em 23 abr 2019]; 19(8). Disponível em: https://doi.org/10.1016/j.cardfail.2013.06.152.

19. Beaton D, Bombardier C, Guillemin F, Ferraz MB. Recommendations for the cross-cultural adaptation of the DASH \& Quick DASH outcome measures. Institute for Work \& Health. 2007 [acesso em 23 abr 2019]; 1. Disponível em: http://dash.iwh.on.ca/sites/dash/files/downloads/cross cultural adaptation 2007.pdf.

20. Polit DF, Beck CT. Fundamentos de Pesquisa em Enfermagem: Avaliação de Evidências para a Prática da Enfermagem. $7^{\circ}$ ed. Porto Alegre; 2016.

21. Alexandre NMC, Coluci MZO. Validade de conteúdo nos processos de construção e adaptação de instrumentos de medidas. Ciênc Saúde Coletiva [Internet]. 2011 [acesso em 23 abr 2019];16(7). Disponível em: http://dx.doi.org/10.1590/S1413-81232011000800006.

22. Paim AE, Nascimento ERP do, Bertoncello KCG, Sifroni KG, Salum NC, Nascimento KC do. Validation of an instrument regarding nursing intervention in patients in vasoactive therapy. Rev. Bras. Enferm [Internet]. 2017 [acesso em 23 abr 2019]; 70(3). Disponível em: http://dx.doi.org/10.1590/0034-71672016-0254.

23. Marinho PML, Campos MP de A, Rodrigues EOL, Gois CFL, Barreto ID de C. Construction and validation of a tool to Assess the Use of Light Technologies at Intensive Care Units. Rev. Latino-Am. Enfermagem [Internet]. 2016 [acesso em 23 abr 2019]; 24. Disponível em: http://dx.doi.org/10.1590/1518$\underline{8345.1002 .2816}$

24. Cunha CM, Neto OP de A, Stackfleth RS. Principais métodos de avaliação psicométrica da confiabilidade de instrumentos de medida. Rev Atenção Saúde [Internet]. 2016 [acesso em 23 abr 
2019];14(49). Disponível em: http://dx.doi.org/10.13037/ras.vol14n49.3671.

25. Matthiensen, A. Uso do coeficiente do alfa de Cronbach em avaliações por questionários. [Internet]. 2011 [acesso em 03 jun 2019]. Disponível em: https://www.infoteca.cnptia.embrapa.br/bitstream/ doc/936813/1/DOC482011ID112.pdf.

26. Ministerio de Salud Pública (UY). Decreto n. 379, de 4 de agosto de 2008, a qual dispõe sobre a Comisión de Bioética y Calidad de Atención, [Internet]. 2008 [acesso em 03 jun 2019]. Disponível em: http://www.impo.com.uy/bases/decretos-originales/379-2008.

27. Riegel B, Lee CS, Dickson WV, Carlson B. An update on the self-care of heart failure index. J Cardiovasc Nurs [Internet]. 2009 [acesso em 23 abr 2019]; 24. Disponível em: http://dx.doi.org/10.1097/ JCN.0b013e3181b4baa0.

28. Riegel B, Carlson B, Moser DK, Sebern M, Hicks FD, Roland V. Psychometric testing of the self-care of heart failure index. J Card Fail [Internet]. 2004 [acesso em 23 abr 2019]; 10(4). Disponível em: https://doi. org/10.1016/j.cardfail.2003.12.001.

29. Barbaranelli C, Lee CS, Vellone E, Riegel B. Dimensionality and reliability of the self-care of heart failure index scales: further evidence from confirmatory factor analysis. research in nursing health. Res Nurs Health [Internet]. 2014 [acesso em 23 abr 2019]; 37(6). Disponível em: http://dx.doi.org/10.1002/ nur.21623.

Recebido: 24/04/2019

Finalizado: 20/09/2019

Autor Correspondente:

Paula Vanessa Peclat Flores

Universidade Federal Fluminense

R. Dr. Celestino, 74 - 24020-091 - Niterói, RJ, Brasil

E-mail: paulapeclat@gmail.com 